\title{
Inferior Vena Cava Syndrome as a Manifestation of Metastatic Carcinoid Tumor
}

\author{
Matthew Stankard ${ }^{\mathrm{a}}$ Erik Soule $^{\mathrm{b}}$ Jerry Matteo ${ }^{\mathrm{b}}$ \\ aDepartment of Radiology, Florida Atlantic University College of Medicine, Boca Raton, FL, USA; \\ ${ }^{b}$ Department of Interventional Radiology, UF Health Jacksonville, University of Florida, Jacksonville, FL, USA
}

\section{Keywords}

Cryoablation · Carcinoid tumor · Inferior vena cava stent · Neuroendocrine tumor · Intrahepatic inferior vena cava

\begin{abstract}
Small bowel-origin carcinoid tumor is indolent but may metastasize relentlessly to various sites, including the liver. Over the past 9 years, we have treated a 69-year-old woman who has undergone 5 percutaneous liver ablations, 5 hepatic intra-arterial chemoembolizations, an ovarian cryoablation, and a trans-ventral hernia mesenteric cryoablation. These interventions are all related to her inoperable carcinoid malignancy. After the patient presented with swelling of the abdomen and both lower extremities, computed tomography (CT) angiography was performed, revealing a circumferential hepatic metastatic mass encasing the intrahepatic inferior vena cava (IVC) and extensive third spacing of fluids specific to the IVC distribution below the diaphragm. A venogram of the intrahepatic IVC revealed extrinsic compression causing $95 \%$ narrowing of the vessel. A balloon was advanced to the level of the lesion and inflated, increasing the caliber of the vessel. Subsequently, 2 covered aortic stent graft cuffs were deployed in an overlapping fashion within the lumen of the IVC, traversing the area of narrowing. Next,
\end{abstract}

an open-cell aortic dissection stent was placed across both overlapping aortic stents from the renal veins to the hepatic veins. Following this, three 17-gauge cryoablation probes were inserted into the segment 1 intrahepatic lesions encasing the newly stented IVC via an anterior percutaneous approach. Two 10-min freeze cycles were performed with intraoperative CT imaging, demonstrating circumferential coverage of the lesions. Posttreatment venogram revealed patent stent grafts within the intrahepatic IVC, and restoration of vessel patency. No immediate postoperative complications were noted. The patient's abdominal and lower extremity swelling resolved completely within 1 week after procedure. Two-month follow-up CT demonstrated markedly decreased size of the metastatic lesions and no adverse effects. Six- and 9-month PET-CT scans demonstrated maintained patency of the IVC stent. This palliative procedure allowed the patient to maintain good performance status and alleviated her symptoms of IVC syndrome. The radial force generated by the multiple aortic stents will ostensibly maintain the patency of the intrahepatic IVC. Cryoablation of the encasing metastatic lesion was performed with markedly decreased size of the tumor on the 2-month follow-up.

C 2021 The Author(s) Published by S. Karger AG, Basel
C 2021 The Author(s)

Published by S. Karger AG, Basel

This article is licensed under the Creative Commons AttributionNonCommercial-NoDerivatives 4.0 International License (CC BYNC-ND) (http://www.karger.com/Services/OpenAccessLicense). Usage and distribution for commercial purposes as well as any distribution of modified material requires written permission.
Correspondence to:

Jerry Matteo,dr_jer@hotmail.com 


\section{Introduction}

Well-differentiated neuroendocrine tumors (NETs) are rare tumors with age-adjusted incidence of nonpancreatic primary tumors falling at approximately 4.7 per 100,000 [1]. Furthermore, the incidence of primary NETs has been steadily rising over time. The age-adjusted incidence rate of primary NETs rose from 1.09 to 6.98 per 100,000 between 1973 and 2012 [2]. This increase is most likely attributable to an increase in detection both on imaging and in endoscopy [3]. NETs tend to arise most frequently in the gastrointestinal tract (55\%) and the bronchopulmonary system (30\%). Within the gastrointestinal tract, NETs are most likely to be found within the small intestine (45\%), especially the ileum, followed by the rectum (20\%), appendix (16\%), colon (11\%), and stomach (7\%) [4].

Histologically, NETs arise from enterochromaffin cells and are classified based on their origin from the embryonic divisions of the alimentary tract: the foregut, midgut, and hindgut. Of these locations, those arising in the midgut (small intestine and proximal colon) produce serotonin that can give rise to the typical carcinoid syndrome. Patients who have a small bowel NET and liver metastasis may experience carcinoid syndrome, although small bowel NETs are more often detected incidentally in asymptomatic patients. If symptoms are present, patients most commonly experience vague, nonspecific abdominal pain and/or intermittent obstruction symptoms as a result of the malignancy [5].

NETs exhibit a predilection for hepatic metastasis, regardless of the site of primary malignancy [6]. Once a tumor has metastasized to the liver, patients often experience a clinical syndrome related to tumor burden that can include generalized abdominal pain, jaundice, abdominal fullness, hepatomegaly, and early satiety. These patients may also experience effects from the release of serotonin into the systemic circulation, referred to as carcinoid syndrome, classic symptoms of which manifest as flushing and diarrhea. In addition to liver metastasis, midgut NETs also tend to metastasize to the mesentery and peritoneum and can cause chronic bowel obstruction, leading to weight loss and malnutrition, intussusception, or ischemia necessitating surgical intervention.

SVC syndrome is often caused by a direct invasion or external compression of the SVC by a malignancy, resulting in a constellation of symptoms including facial plethora, jugular venous distension, and upper extremity swelling that occurs as a result of compromised blood flow through the SVC [7]. Malignant compression of the inferior vena cava (IVC) however causing a similar clinical syndrome is much less frequently seen. In this article, we present the case and treatment of a patient with known history of metastatic small bowel-origin NET. The patient presented with abdominal and bilateral lower extremity swelling and was found to have computed tomography (CT) evidence for a circumferential hepatic metastasis that encased the intrahepatic IVC (Fig. 1a, b) and caused extensive IVC distribution third spacing of fluids (Fig. 2a, b).

\section{Case Report}

The patient described in this article was not a surgical candidate owing to her metastatic diagnosis and large ventral hernia. She presented with complications of a metastatic tumor encasing and obstructing flow through the IVC. This had caused debilitating lower abdominal and bilateral lower extremity swelling. Urgent stenting of the IVC using aortic stent grafts was performed.

Using a 21-guage needle and ultrasound guidance, the right internal jugular vein was accessed, and a guide wire was introduced into the IVC. Over the guide wire, a 5-French pigtail catheter was advanced into the intrahepatic IVC. Following this, using a 21-guage needle and ultrasound guidance, the right common femoral vein was accessed and a guide wire was introduced into the IVC. Under fluoroscopic guidance, a micropuncture sheath was exchanged first for a 9-French sheath, followed by an 18-French sheath.

Through the pigtail catheter, an initial IVC venogram was performed, which demonstrated severe stenosis of the IVC at the level of the lower intrahepatic portion (Fig. 3a). The bilateral renal veins were noted at the level of the superior endplate of L2. The bilateral hepatic veins were located at the level of the inferior endplate of T11.

Next, through the right common femoral vein sheath and over a guide wire, a covered aortic cuff stent $(28 \times 33 \mathrm{~mm})$ was advanced and positioned along the inferior margin of the stenosis. Once satisfactory positioning was achieved, the stent was deployed. Angioplasty using a compliant CODA balloon (Gore, Flagstaff, AZ, USA) was performed over the region of greatest stenosis. This demonstrated improvement; however, there was still significant residual stenosis. Therefore, the decision was made to place an additional stent using the same technique with an additional covered stent $(28 \times 33 \mathrm{~mm})$ that was advanced and positioned so that the superior edge was below the level of the hepatic veins. Angioplasty was again performed with a compliant CODA balloon (Gore, Flagstaff, AZ, USA) over the region of greatest stenosis. After stenting and angioplasty, venogram done at this point demonstrated significantly improved luminal diameter (shown in Fig. 3b).

In order to better preserve the luminal diameter due to extrinsic compression from the intrahepatic tumors, an additional opencell stent $(36 \times 80 \mathrm{~mm})$ (Cook Medical, Bloomington, IN, USA) was advanced through the right common femoral vein sheath and positioned so that the superior end was approximately $1.5 \mathrm{~cm}$ below the inferior cavoatrial junction $(36 \times 36 \times 80 \mathrm{~mm})$. Once satisfactory positioning had been achieved, the stent was deployed. 
Fig. 1. a Axial contrast-enhanced CT image demonstrating diffuse hypoattenuating liver metastases (red arrows). b Coronal contrast-enhanced CT image demonstrating 2 metastases, seen to exert marked mass effect on the IVC (yellow arrows). IVC, inferior vena cava; $\mathrm{CT}$, computed tomography.

Fig. 2. a Axial contrast-enhanced CT image at the level of the heart. Subcutaneous soft tissue above the diaphragm is unremarkable (red arrows). b Axial contrast-enhanced CT image at the level of the sacroiliac joints demonstrating diffuse soft tissue infiltration below the diaphragm (yellow arrows). CT, computed tomography.

Fig. 3. a-c IVC venograms which demonstrate (a) severe external compression (red arrows), (b) stent deployment (blue arrows), and (c) post-stenting and angioplasty with improved patency of the IVC (yellow arrows). IVC, inferior vena cava.

Fig. 4. a, b Axial and coronal contrast-enhanced CT images demonstrating patent stents within a widely patent IVC (red arrows). IVC, inferior vena cava; CT, computed tomography.
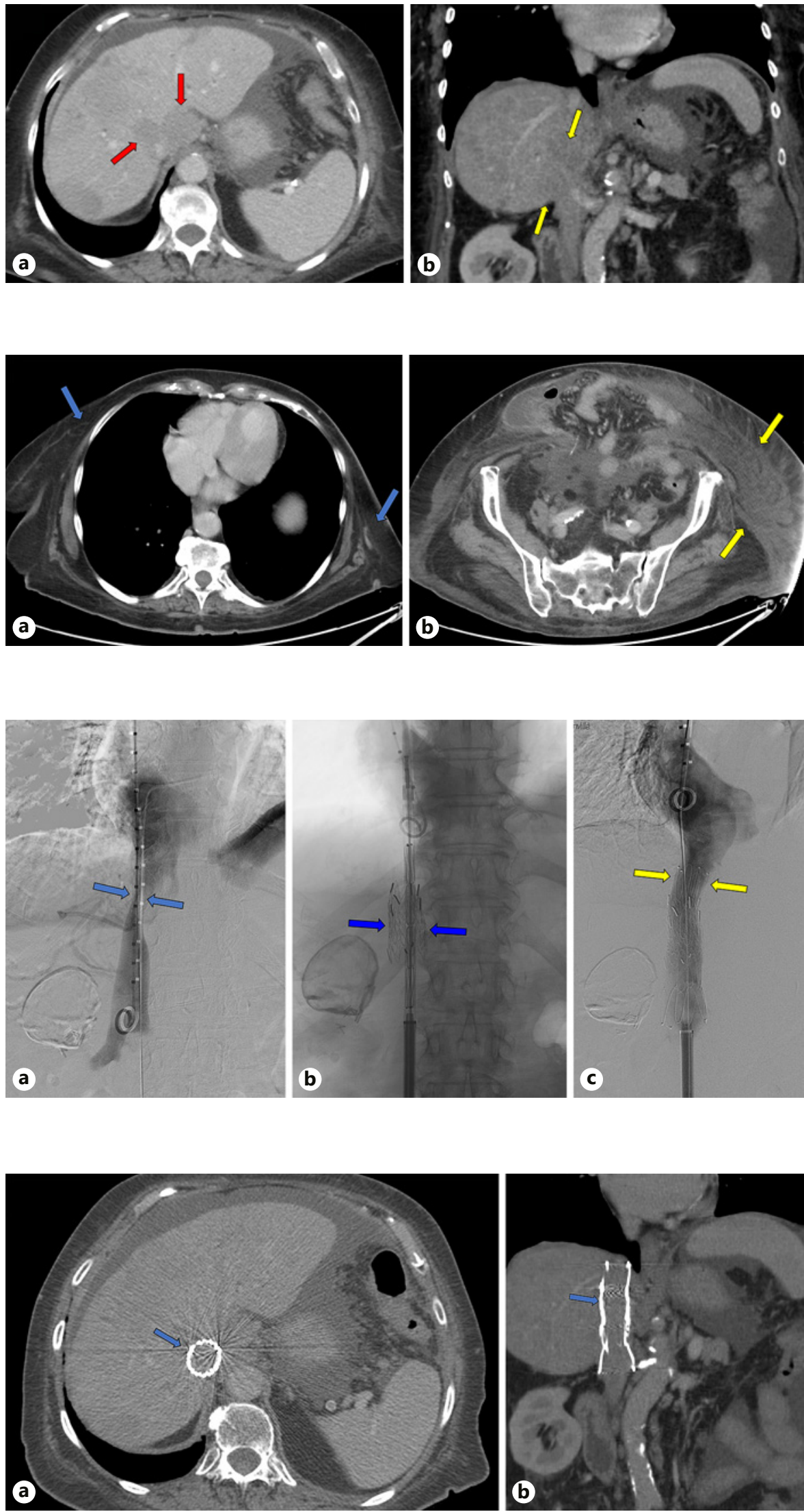

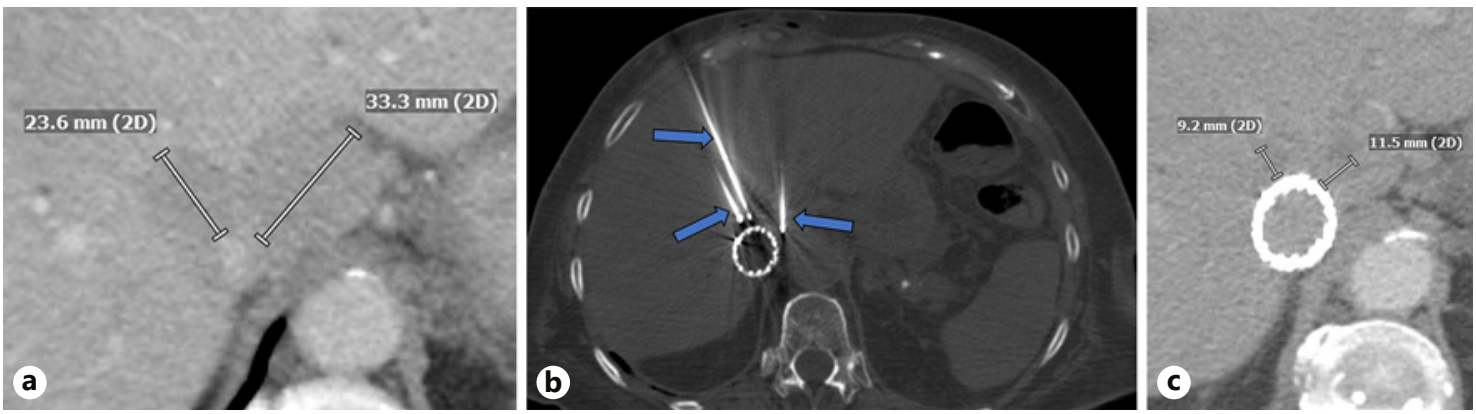

Fig. 5. a Axial contrast-enhanced CT images before cryoablation. b Axial intraoperative CT image demonstrating cryoablation probes within the previously noted segment I metastatic lesions (red arrows). c Postcryoablation 2- month follow-up demonstrating reduction in the size of segment I metastatic lesions and patent stent within the IVC. IVC, inferior vena cava; CT, computed tomography.
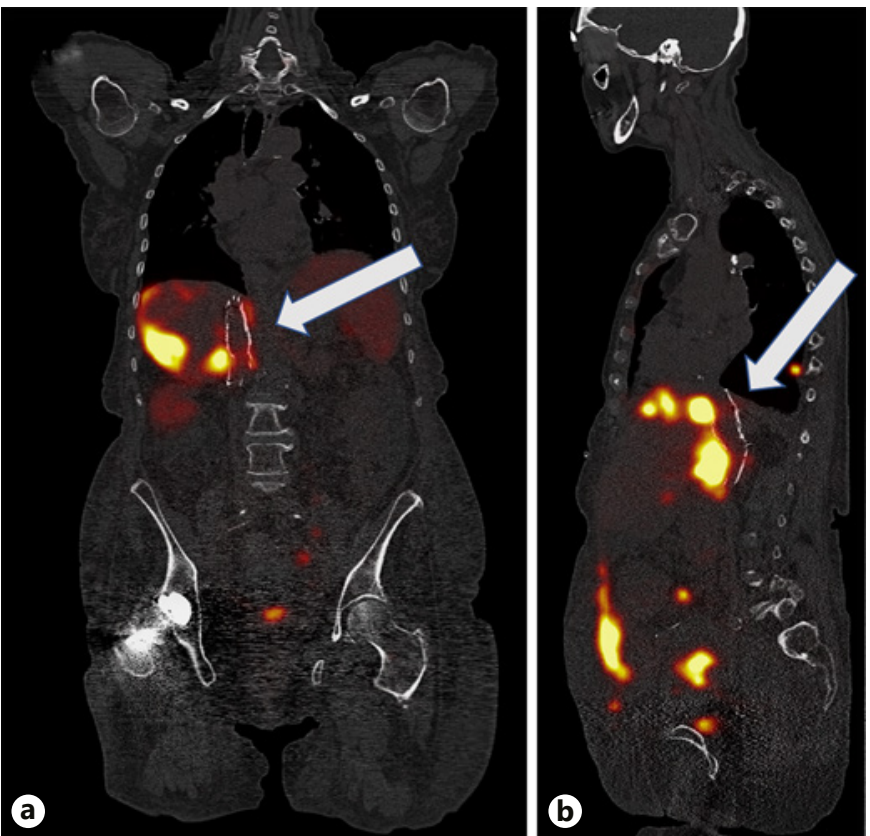

Fig. 6. a Coronal Ga68 Dotatate PET-CT scan showing maintenance of IVC stent patency at 6-month follow-up (white arrow). b Sagittal Ga68 Dotatate PET-CT scan showing maintenance of IVC stent patency at 6-month follow-up (white arrow). IVC, inferior vena cava; CT, computed tomography.

Angioplasty utilizing the 37-mm molding and an occlusion balloon was performed over the regions of greatest stenosis. After stenting and angioplasty, venogram demonstrated significantly improved luminal diameter with no stenosis, and flow to the heart (shown in Fig. 3c). Nonenhanced CT of the abdomen showed the widely patent IVC stents (Fig. 4a, b).

Approximately 6 weeks later, the patient returned for cryoablation. Limited contrast-enhanced CT images were performed to delineate the 3 liver masses for procedure planning (Fig. 5a). Using CT guidance, a total of three 17 -gauge cryoablation probes were
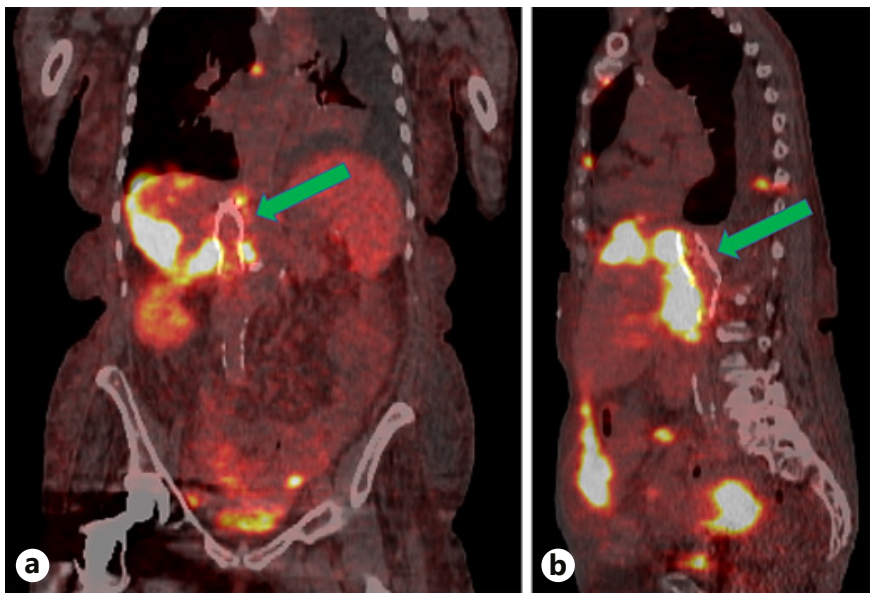

Fig. 7. a Coronal Ga68 Dotatate PET-CT scan showing maintenance of IVC stent patency at 9-month follow-up (green arrow). b Sagittal Ga68 Dotatate PET-CT scan showing maintenance of IVC stent patency at 9-month follow-up (green arrow). IVC, inferior vena cava; CT, computed tomography.

inserted into the liver masses using direct CT fluoroscopy. The most medial probe was positioned in the superior portion of the caudate immediately adjacent to the IVC and aorta. The more lateral 2 were positioned to progress along the lateral aspect of the IVC and the pericaval mass (Fig. 5b). Specifically, the tract of these lateral 2 probes were planned such that they would traverse the third lesion, located more superficially in the right lobe of the liver, along their retraction tract.

Limited CT images were obtained at 5-and 10-min intervals to evaluate the progression of the ice balls with demonstrated circumferential coverage of the first 2 liver masses. The most medial probe was then removed. The 2 lateral probes were retracted approximately $4 \mathrm{~cm}$, and the third lesion was treated. Limited CT images were obtained at 5-and 10-min intervals. Following removal of the lateral 2 cryoablation probes, limited CT was performed, which revealed hypodensities within the region of the liver masses consistent with ice balls. 
No complications or injury to surrounding organs was noted. The patient tolerated the procedure well without immediate complications. The abdominal and lower extremity swelling resolved completely within 1 week after the procedure. Not only was this treatment modality effective in the short term but the stents maintained patency until cryoablation of the offending lesion could be performed approximately 6 weeks later. Stent patency was again demonstrated on repeat IVC venogram 8 months later when the patient presented with bilateral lower extremity swelling. IVC venogram at that time demonstrated wide patency of the IVC stents with low intravascular volume. The new onset edema was determined to be due to liver failure and poor nutritional status, resulting in hypoalbuminemia, unrelated to stenting.

Cryoablation of the metastatic lesion was performed with markedly decreased size of the tumor demonstrated on follow-up CT 2 months later (Fig. 5c). There were no adverse outcomes reported in the immediate postoperative period or on follow-up over the next 9 months. Follow-up Ga68 Dotatate PET-CT scans performed at 6 (Fig. 6a, b) and 9 months (Fig. 7a, b) demonstrated maintained patency of the stent.

\section{Discussion}

NETs demonstrate a strong propensity for metastasis with $82 \%$ of NETs showing liver metastasis at autopsy [8]. This case demonstrates a rare case of IVC syndrome seen in a patient with metastatic small bowel NET. These results are consistent with results seen in Lee et al. [9], which described long-term efficacy of IVC stent placement in which 14 patients underwent stent placement following liver transplant. Zhang et al. [10] also saw both high levels of technical success in the placement of IVC stents in Budd-Chiari syndrome and long-term patency with $96.7 \%$ of IVC stents remaining patent over a mean followup time of 49 months.

Cryoablation has been used in the treatment of primary and metastatic liver tumors in cases where surgical excision is contraindicated, such as in the case of vascular invasion, metastatic disease, poor liver reserve, or multifocal disease. Cooling down to $-35^{\circ} \mathrm{C}$ is required to achieve a reliable tumor cell kill [11]. Cell death however is not a direct consequence of lowering tissue temperature but is caused by destruction of cellular architecture secondary to ice crystal formation during rapid freezing [11]. This cellular destruction occurs through multiple mechanisms including protein denaturation, osmosisdriven shifts of intracellular and extracellular water, membrane destabilization, cellular rupture, and tissue ischemia [12]. Following one freeze-thaw cycle, approximately $10 \%$ of cancer cells are still viable; however, when a minimum of 2 cycles are used, approximately $100 \%$ of tumor cells are rendered nonviable [13].
Types of tumors amenable to treatment with hepatic cryoablation include hepatocellular, colorectal, neuroendocrine, Wilms', renal cell, and adrenocortical tumors [13]. Percutaneous thermal techniques such as radiofrequency ablation, microwave ablation, and percutaneous cryoablation (PC) are often employed in these cases and have been shown to have long-term results similar to surgical resection [14]. PC however may be relatively contraindicated in certain cases due to the "thermal sink" effect [15]. This effect refers to the effect of flowing blood in major vessels near the site of cryoablation, creating difficulty in ice ball formation due to the thermal flow of heat in the blood adjacent to the lesion. As large volumes of blood pass by the lesion, continual thermal energy is delivered to the ablation site, disrupting ice ball formation. This can lead to incomplete ablation and tumor recurrence [16]. This phenomenon has been overcome by the use of an intermittently inflated angioplasty balloon within the IVC, causing temporary cessation of blood flow [16].

The five-year survival for patients with metastatic carcinoid tumor is 19-38\% [17]. Recent advances in minimally invasive modalities have reduced the need to surgically resect these tumors [8]. Our PC results are consistent with those in the literature which shows PC to be technically effective at halting local tumor growth in $89.5 \%$ of lesions over a 3-month period [18]. Minimally invasive modalities such as PC have been instrumental in prolonging survival in these patients.

IVC syndrome may be considered as an etiology of new-onset lower extremity swelling in patients with history of primary or metastatic hepatic malignancy. This case demonstrates the efficacy of IVC stenting to maintain patency, as well as its value as a bridge to more definitive treatment. Minimally invasive PC can be considered for poor surgical candidates and can be performed adjacent to a stent graft in the intrahepatic IVC.

\section{Statement of Ethics}

Informed, written consent was obtained from the patient prior to participation in this study. This case was reviewed by UF IRB-03 chairman, and it was determined that as a single case, it does not require IRB approval.

\section{Conflict of Interest Statement}

The authors have no conflicts of interest to declare. 


\section{Funding Sources}

There are no funding sources to disclose.

\section{Author Contributions}

All of the listed authors either worked on the manuscript or figures presented.

\section{References}

1 Yao JC, Hassan M, Phan A, Dagohoy C, Leary C, Mares JE, et al. One hundred years after "carcinoid": epidemiology of and prognostic factors for neuroendocrine tumors in 35,825 cases in the United States. J Clin Oncol. 2008; 26(18):3063-72.

2 Dasari A, Shen C, Halperin D, Zhao B, Zhou $\mathrm{S}, \mathrm{Xu} \mathrm{Y}$, et al. Trends in the incidence, prevalence, and survival outcomes in patients with neuroendocrine tumors in the United States. JAMA Oncol. 2017 Oct 1;3(10):1335-42.

3 Modlin IM, Champaneria MC, Chan AK, Kidd M. A three-decade analysis of 3,911 small intestinal neuroendocrine tumors: the rapid pace of no progress. Am J Gastroenterol. 2007 Jul;102(7):1464-73.

4 Maggard MA, O’Connell JB, Ko CY. Updated population-based review of carcinoid tumors. Ann Surg. 2004 Jul;240(1):117-22.

5 Saha S, Hoda S, Godfrey R, Sutherland C, Raybon K. Carcinoid tumors of the gastrointestinal tract: a 44-year experience. South Med J. 1989;82(12):1501-5.

6 Strosberg J, Gardner N, Kvols L. Survival and prognostic factor analysis of 146 metastatic neuroendocrine tumors of the mid-gut. Neuroendocrinology. 2009 Jun;89(4):471-6.
7 Rice TW, Rodriguez RM, Light RW. The superior vena cava syndrome: clinical characteristics and evolving etiology. Medicine. 2006 Jan;85(1):37-42.

8 Soule E, Bagherpour A, Matteo J. Freezing Fort Knox: mesenteric carcinoid cryoablation. Gastrointest Tumors. 2017;4(1-2):5360.

9 Lee JM, Ko GY, Sung KB, Gwon DI, Yoon HK, Lee SG. Long-term efficacy of stent placement for treating inferior vena cava stenosis following liver transplantation. Liver Transpl. 2010;16(4):513-9.

10 Zhang CQ, Fu LN, Xu L, Zhang GQ, Jia T, Liu JY, et al. Long-term effect of stent placement in 115 patients with Budd-Chiari syndrome. World J Gastroenterol. 2003;9(11):2587-91.

11 Pearson AS, Izzo F, Fleming RY, Ellis LM, Delrio P, Roh MS, et al. Intraoperative radiofrequency ablation or cryoablation for hepatic malignancies. Am J Surg. 1999 Dec 1; 178(6):592-9.

12 Bayjoo P, Jacob G. Hepatic cryosurgery: biological and clinical considerations. J R Coll Surg Edinb. 1992 Dec;37(6):369-72.
13 Iannitti DA, Heniford T, Hale J, GrundfestBroniatowski S, Gagner M. Laparoscopic cryoablation of hepatic metastases. Arch Surg. 1998 Sep 1;133(9):1011-5.

14 Nault JC, Sutter O, Nahon P, Ganne-Carrié N, Séror O. Percutaneous treatment of hepatocellular carcinoma: state of the art and innovations. J Hepatol. 2018;68:783-97.

15 Soule E, Lamsal S, Lall C, Matteo J. Eye opener to $\mathrm{EtOH}$ ablation for juxta-cardiac hepatocellular carcinoma. Gastrointest Tumors. 2018;5(3-4):109-16.

16 Soule E, Matteo J. Finally, a minimally invasive option for intrahepatic inferior vena cava invasion by hepatocellular carcinoma. Gastrointest Tumors. 2018;5(1-2):54-61.

17 Riihimäki M, Hemminki A, Sundquist K, Sundquist J, Hemminki K. The epidemiology of metastases in neuroendocrine tumors. Int J Cancer. 2016 Dec 15;139(12):2679-86.

18 Glazer DI, Tatli S, Shyn PB, Vangel MG, Tuncali K, Silverman SG. Percutaneous imageguided cryoablation of hepatic tumors: single-center experience with intermediate to long-term outcomes. AJR Am J Roentgenol. 2017 Dec 1;209(6):1381-9. 